\title{
Peripheral medulloepithelioma: a rare tumor with a potential target therapy
}

\author{
Maria Debora De Pasquale ${ }^{1 *}$, Maria Antonietta De loris ${ }^{1}$, Angela Gallo ${ }^{1}$, Angela Mastronuzzi ${ }^{1}$, Alessandro Crocoli ${ }^{2}$, \\ Raffaele Cozza ${ }^{1}$ and Renata Boldrini ${ }^{3}$
}

\begin{abstract}
Background: Medulloepithelioma (ME) is a rare embryonal tumor predominantly located in the eye or in the central nervous system without an established treatment.

Case presentation: We report of a case of a localized peripheral ME treated with conventional and high dose chemotherapy, surgery and local radiotherapy. At relapse, the tumor tissue revealed a different molecular signature compared to the initial tumor mass. This molecular signature revealed a high expression of platelet derived growth factor receptor (PDGFR). Sorafenib plus irinotecan and temozolomide was started with a 5 month progression free survival.

Conclusion: Our experience suggests a possible role of sorafenib or different PDGFR inhibitors in ME. Targeting treatment could represent an adjuvant and/or alternative therapy for ME and other rare tumors.
\end{abstract}

Keywords: Peripheral medulloepithelioma, Treatment, Target therapy, PDGF

\section{Background}

Medulloepithelioma (ME) is a rare embryonal tumor with a distinctive pathology characterized by papillary and tubular patterns recalling the primitive epithelium of the medullary plate and the embryonal neural tube [1].

ME is usually located in the eye or in central nervous system (CNS); a peripheral location has been rarely reported. Intraocular ME is often a well-circumscribed and benign tumor, while CNS ME is an aggressive neoplasm associated with a good prognosis only if completely removed $[2,3]$. Considering the rarity of peripheral medulloephitelioma (PME), the optimal treatment has yet to be established.

We report a case of PME with an interesting target protein expression suggesting a possible alternative therapeutic strategy for this rare tumor.

\section{Case presentation}

A 3 year old female presented with a one-month history of abdominal pain and anorexia. The abdominal

\footnotetext{
* Correspondence: mdebora.depasquale@opbg.net

'Department of Pediatric Hematology/Oncology and Stem Cell Transplantation, Bambino Gesù Children's Hospital, Piazza Sant'Onofrio, 4 - 00165 Rome, Italy

Full list of author information is available at the end of the article
}

ultrasonography showed a retroperitoneal mass $(7 \times 6 \times$ $6 \mathrm{~cm}$ ) confirmed by CT scan with a right hydronephrosis without evidence of metastatic spread (Figure 1A). An open biopsy of the lesion was performed. The pathology revealed a malignant neoplasm composed of tubules, papillary structures, ribbons of primitive stratified columnar cells, vesicular nuclei, and high nuclear-cytoplasmic ratio. This histopathology is similar to the structure of the primitive epithelium of the medullary plate and neural tube. The absence of cilia and blepharoplasts ruled out the hypothesis of a papillary ependymoma. The neoplastic cells showed a diffuse positivity for CD56 and WT1 (cytoplasmic) and a variable positivity for NSE, Synaptophisin, S100 protein and Cytokeratin MNF116, while they were negative for CD99, alpha-fetoprotein, CD30, OCT3/4, $\beta$-HCG. The diagnosis was neuroectodermal embryonal tumor with patterns of ME (Table 1, Figure 2 and Figure 3).

The child started chemotherapy according to our local protocol for Ewing Sarcoma Family Tumor [4]. After 2 ICE courses (Ifosfamide $2 \mathrm{gr} / \mathrm{m}^{2}$ for 3 days, Carboplatin $400 \mathrm{mg} / \mathrm{m}^{2}$ for 2 days and Etoposide $150 \mathrm{mg} / \mathrm{m}^{2}$ for 3 days) and 2 CAV (Cyclophosphamide $1.5 \mathrm{mg} / \mathrm{m}^{2}$ for 2 days, Vincristine $0.5 \mathrm{mg} / \mathrm{m}^{2}$ for 3 days and Doxorubicin $25 \mathrm{mg} / \mathrm{m}^{2}$ for 3 days), she achieved partial response, the mass measuring $5 \times 3.3 \times 3.8 \mathrm{~cm}$ (Figure 1B). Grade 4 


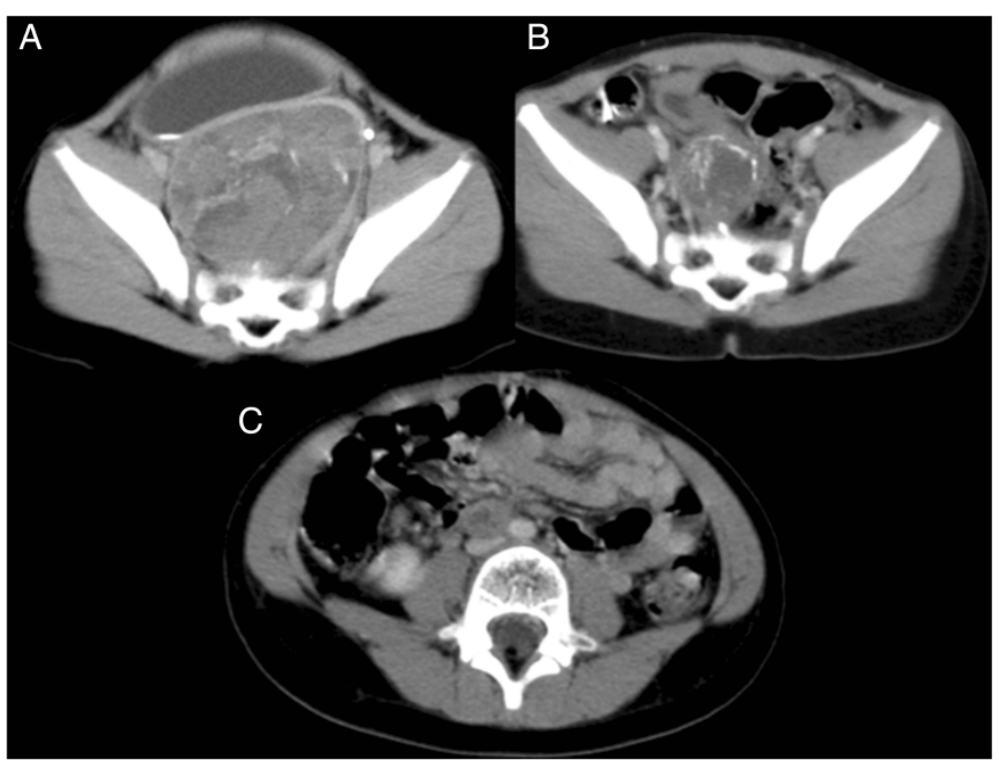

Figure $1 \mathrm{~A}$ : $\mathrm{CT}$ scans showing the tumor at diagnosis right hydronephrosis is also present; scans after 4 courses of $C T$, demonstrating partial response to therapy; C: recurrence of the tumor after six months from stop therapy. $C$ t at diagnosis (A), after chemotherapy (B) and at relapse (C).

bone marrow toxicity that required red blood cells and platelets transfusion and hospitalization for neutopenic fever, was recorded after all courses.

A complete resection of the lesion was performed. The pathology showed extensive involutive post-chemotherapy aspects. The residual viable tumor showed histologic aspects overlapping with these of the first biopsy, partly characterized by more solid areas, with the same immunophenotypic pattern (Figure 4).

\section{Table 1 Tumor immunochemistry at diagnosis and at} relapse

\begin{tabular}{llc}
\hline Immunochemistry & Diagnosis & Relapse \\
\hline CD56 & ++ & ++ \\
WT1 & ++ (cytoplasmatic) & ++ \\
NSE & +- & +- \\
Synaptophisin & +- & +- \\
S100 protein & +- & +- \\
Cytokeratin MNF116 & +- & +- \\
CD99 & - & - \\
Alpha-fetoprotein & - & - \\
CD30 & - & - \\
OCT 3/\$ & - & - \\
B-HCG & - & - \\
EGFR & - & ++ \\
PDGFR & + & - \\
ADAR 1 & + & + \\
ADAR 2 & - & - \\
\hline SEEN EGF & - & -
\end{tabular}

LEGEND: EGFR, epidermal growth factor receptor; PDGFR, platelet-derived growth factor receptor; ADAR, adenosine deaminases that act on RNA.
The child, in complete remission, completed the treatment with two CE courses (Cyclophosphamide $1.5 \mathrm{gr} / \mathrm{m}^{2}$ for 2 days and Etoposide $150 \mathrm{mg} / \mathrm{m}^{2}$ for 2 days) and a last CAV course. As a consolidation treatment, she received a high-dose chemotherapy based on Busulfan and Melphalan with autologous peripheral blood stem cells rescue and, finally, radiotherapy to the primary tumor bed (19, 5 Gy). During the entire chemotherapy treatment, only grade IV bone marrow toxicity was recorded. During radiotherapy the patient presented only grade I diarrhea.

Six months after treatment discontinuation, she presented with an abdominal relapse (Figure 1C). Surgery was performed, achieving a second complete remission. The pathology confirmed a ME with the same characteristics of the primary tumor. At relapse, expression of tumor target proteins was evaluated on tissue specimens obtained both at diagnosis and at recurrence. Immunohistochemistry showed the tumor cells always negative for epidermal growth factor receptor (EGFR). By contrast, there was some positive staining with platelet-derived growth factor receptor (PDGFR) in the primary specimen, with a few cellular clusters showing cytoplasmic positivity, while at recurrence a clear diffuse cytoplasmatic positivity for PDGFR reaching almost $100 \%$ of the cells was observed. ADAR2 (adenosine deaminases that act on RNA) was absent in both primary and recurrent tumors; ADAR1 was expressed in the nucleus and cytoplasm, both at diagnosis and at recurrence. Figure 5 shows the EGFR, PDGFR, ADAR 1 and ADAR2 expression in tumor specimen at diagnosis and at recurrence. (See also Table 1).

According to the target protein expression, the patient started sorafenib $200 \mathrm{mg}$ once daily orally, plus temozolomide 


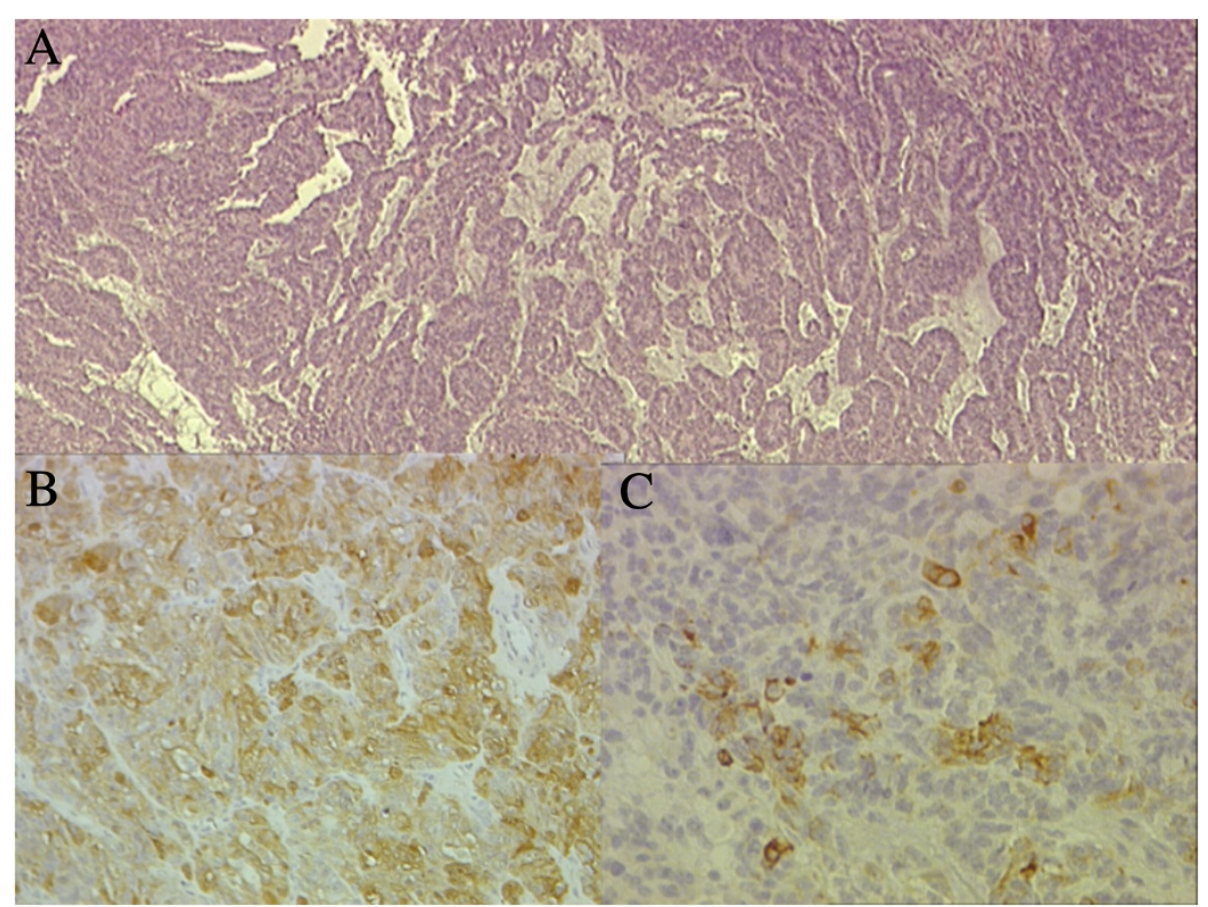

Figure 2 Pathology of the tumor at diagnosis. Papillary and tubular patterns represent the distinctive appearance of medulloepithelioma; HE $4 \times$ (A). Positivity of the neoplastic cells for S100 protein 60× (B) and for PanCytokeratin 60× (C).

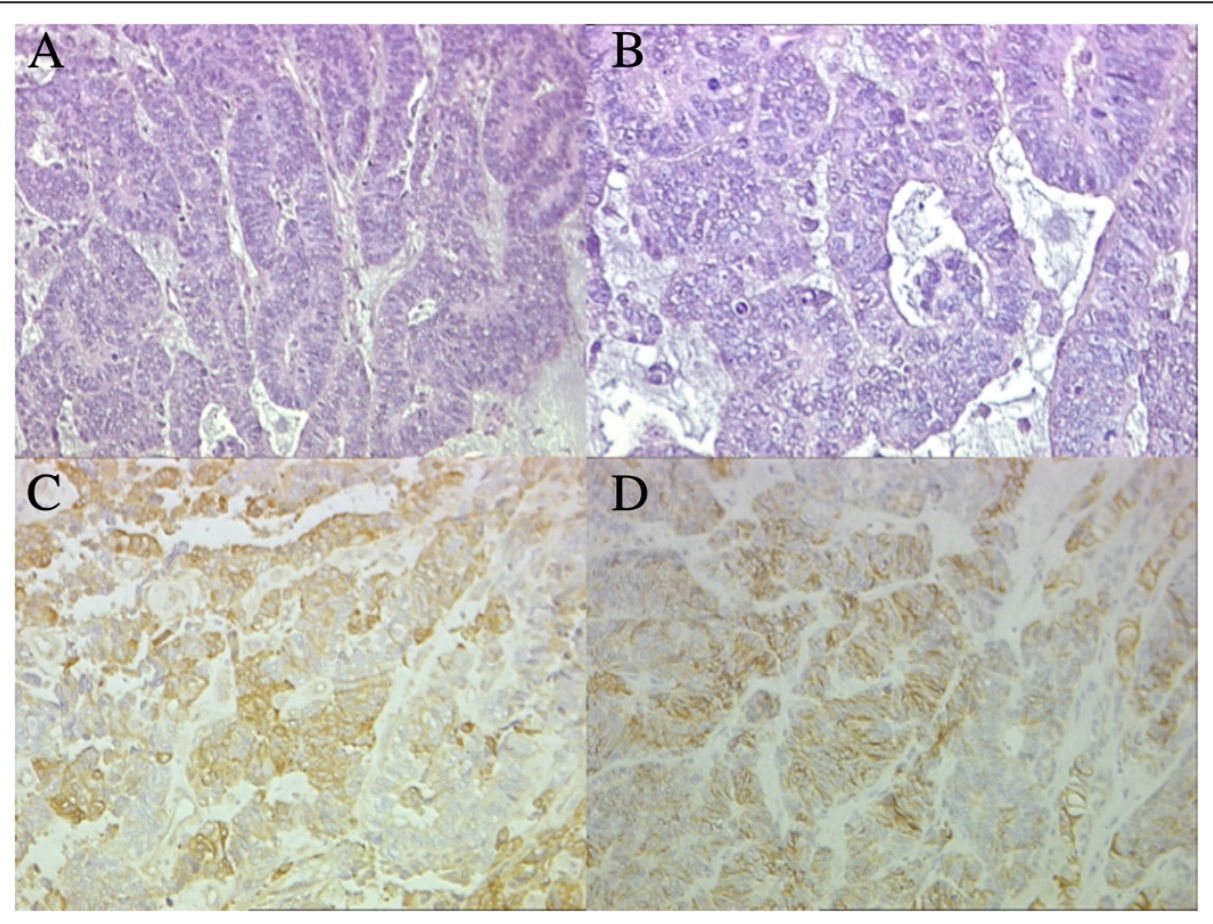

Figure 3 Pathology of the tumor at diagnosis. Areas of tumor at higher magnification show stratified columnar cells bordered by internal and external limiting membrane HE 40× (A) and 60× (B). Positivity of the neoplastic cells for NSE 60× (C) and for CD56 60× (D). 


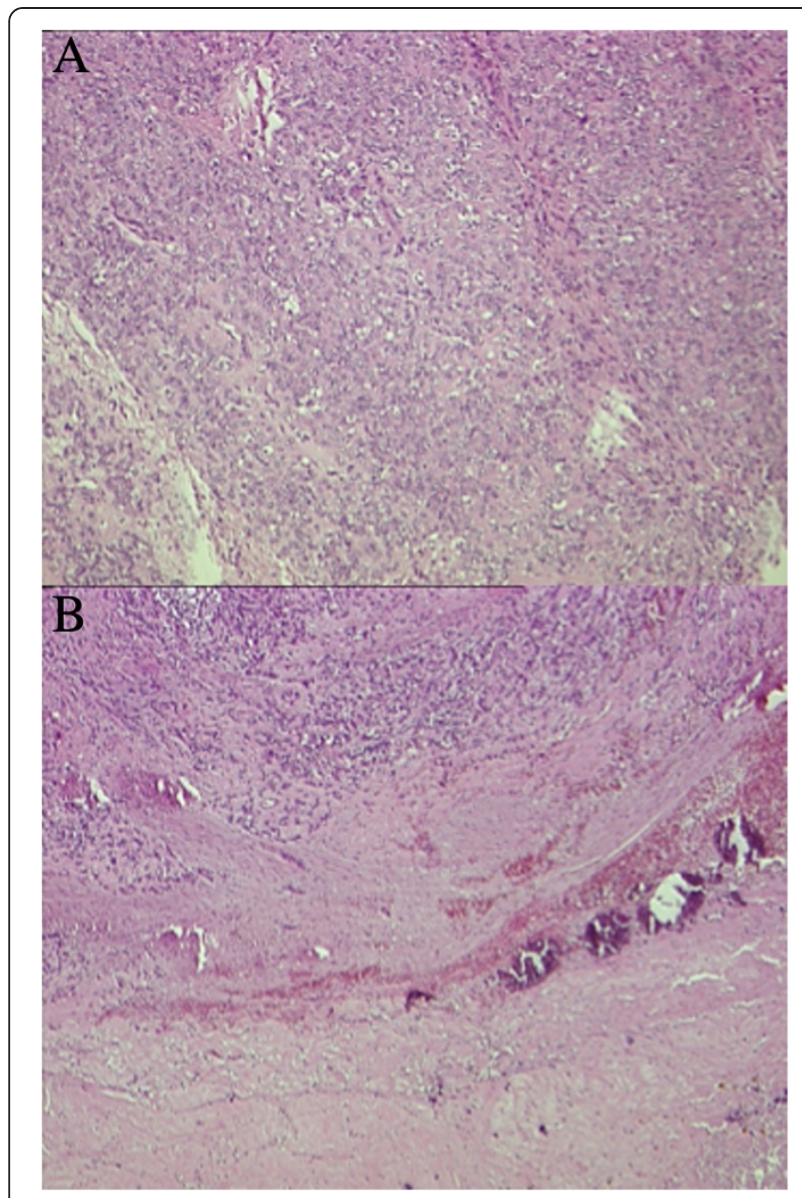

Figure 4 Pathology after chemotherapy. The post chemotherapy tumor shows extensive involution area, calcifications HE $4 \times(\mathbf{A})$ and a predominant solid pattern $4 \times$ (B).
$100 \mathrm{mg} / \mathrm{m}^{2} /$ day orally for five consecutive days and irinotecan $10 \mathrm{mg} / \mathrm{m}^{2} /$ day orally for 14 consecutive days; the course was repeated every 28 days.

Treatment was well tolerated, only generalized skin rash associated with grade I dry skin not requiring treatment discontinuation was recorded. A progression-free survival was achieved for five months when peritoneal carcinomatosis with an important neoplastic peritoneal effusion appeared. The patient died for progressive disease a few weeks later.

\section{Discussion}

PME is a very rare neoplasm and only few reports describe this entity (see Table 2 for more details) [5-9]. Similar to classical ME, surgery in PME, with or without systemic chemotherapy and/or local radiotherapy, represents a therapeutic option. Complete surgery seems to be associated with better outcome [5-9]. All reported PME were located in the pelvic cavity (Table 2). Some authors hypothesized that these tumors originated from undifferentiated cells of the pre-sacral remnant [6]. Four patients with ovarian ME had a good prognosis after surgery either alone or associated with adjuvant chemotherapy and/or radiotherapy [5]. In a case of congenital pelvic ME, prolonged disease-free survival was achieved after partial surgical removal and chemotherapy [7]. In a similar case, pelvic ME was resistant to chemotherapy and the patient died of metastatic pulmonary progression [6].

Analyzing the reported cases, it seems that some PME with favorable prognosis were sensitive to chemotherapy,

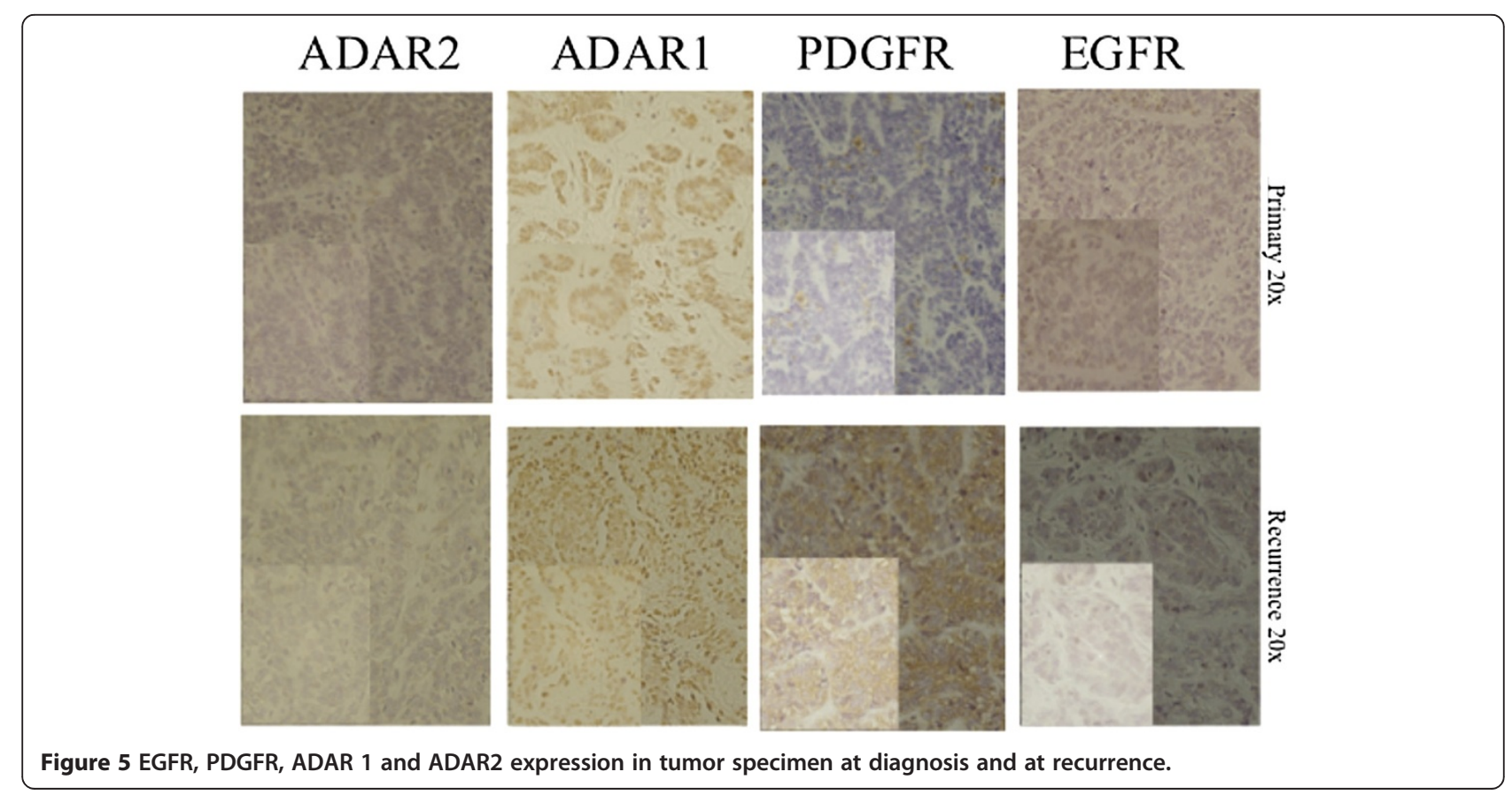


Table 2 Review of literature for peripheral ME

\begin{tabular}{|c|c|c|c|c|c|c|c|}
\hline Reference & Sex & Age & Site & Stage & Metastasis & Therapy & Outcome \\
\hline Kleinman et al. & $\mathrm{F}$ & $20 \mathrm{yr}$ & ovary & I & No & Complete surgery, CT & NED, $108 \mathrm{mo}$ \\
\hline Kleinman et al. & $\mathrm{F}$ & $32 \mathrm{yr}$ & ovary & I & No & Complete surgery, CT & NED, $36 \mathrm{mo}$ \\
\hline Kleinman et al. & $\mathrm{F}$ & $13 \mathrm{yr}$ & ovary & III & No & Complete surgery & DOD, $20 \mathrm{mo}$ \\
\hline Kleinman et al. & $\mathrm{F}$ & $23 \mathrm{yr}$ & ovary & III & No & Complete surgery, CT, RT & $\mathrm{DOD}, 2 \mathrm{mo}$ \\
\hline Figarella-Branger et al. & $\mathrm{F}$ & $17 \mathrm{yr}$ & pelvis & IV & Lung & Surgery on primitive tumor, $C T$ & $\mathrm{DOD}, 8 \mathrm{mo}$ \\
\hline Bruggers et al. & $\mathrm{F}$ & Newborn & pelvis & III & No & Partial resection, CT & NED, $50 \mathrm{mo}$ \\
\hline Donner et al. & F & $12 \mathrm{yr}$ & pelvis & III & No & $\mathrm{CT}$ & NED, 36 mo \\
\hline Nakamura et al. & M & $6 \mathrm{mo}$ & pelvis (sciatic nerve) & III & No & Complete surgery & NED, 84 mo \\
\hline
\end{tabular}

Legend: $F$, female; $M$, male; yr, years; mo, months; $C T$, chemotherapy; RT, radiotherapy; NED, non evidence of disease; DOD, died of disease.

while, for chemo-resistant tumors, the only curative treatment was represented by radical surgery [5-9]. In our case, we decided to treat the patient with an aggressive first-line therapy. The patient achieved complete remission but she relapsed only six months after the end of treatment. At relapse we evaluated the expression of tumor target proteins focusing on the protein expression profile often involved in brain cancers with possible therapeutic implications, such as PDGFR and EGFR. The PDGFR is a cell surface receptor linked to a tyrosine kinase (TK) involved in several processes, including autocrine cancer cells growth and angiogenesis. Few PDGFR antagonists have been developed and introduced in clinical practice, such as sorafenib or STI571/imatinib mesylate [10]. Similarly, EGFR is a cell-surface receptor involved in DNA synthesis and cell migration, adhesion and proliferation. Anticancer drugs directed against EGFR include gefitinib, erlotinib and cetuximab [11].

We also studied the ADARs (adenosine deaminases that act on RNA) expression on tumor specimens. The ADARs are enzymes responsible for Adenosine-to-Inosine conversion on coding and non-coding RNA (such as microRNA) that are emerging as important key proteins in cancers. Recent evidences have connected ADARs (ADAR1 and ADAR2) deregulation to several cancers [12].

Surprisingly, at recurrence, we observed PDGFR expression, not present at diagnosis, in almost all tumor cells.

Sorafenib is a small molecular inhibitor of several TK protein, such as vascular endothelial growth factor receptor, PDGFR and Raf kinases (more avidly C-Raf than B-Raf). The drug has been approved by the U.S. Food and Drug Administration for use in the treatment of advanced renal cancer, unresectable hepatocellular carcinoma and locally recurrent or metastatic, progressive differentiated thyroid carcinoma refractory to radioactive iodine treatment [13-15]. Some authors showed that sorafenib blocks STAT3 (a signal transducer and activator of transcription), as well as the expression of proteins regulating the cell cycle and the apoptosis process, both in cell lines and primary tumor cells of medulloblastoma. These findings provide a rationale for treatment of pediatric medulloblastoma with sorafenib [16]. ME is classified in the group of the embryonal tumors together with medulloblastoma according to WHO classification.

At recurrence, we proposed, as compassionate treatment, sorafenib plus temozolomide and irinotecan. After obtaining IRB approval the three drug combination was started. Treatment was well tolerated and only a mild skin rash was observed.

Sorafenib was chosen according to the PDGFR expression on tumor specimen, while temozolomide and irinotecan had demonstrated activity in medulloblastoma $[17,18]$. We anticipated an efficacy of this drug combination with a good tolerance and a good quality of life considering the oral assumption.

The finding of poor expression of PDGFR at diagnosis and its massive expression at relapse could suggest that a cell clone with high expression of PDGFR was responsible for the relapse. This leads us to hypothesize that sorafenib, if it had been administered at diagnosis, could have allowed to maintain a longer complete remission.

\section{Conclusion}

Our experience is only a single report, with obvious anecdotal consideration. However this report may suggest that in cases of ME the target protein expression in tumor tissue should be evaluated aimed to identify possible therapeutic targets. Moreover, a possible therapeutic role of PDGFR inhibitors was never reported before.

A TK-inhibitor could be considered and employed also during first line treatment. Indeed, only a multicentric trial could asses the clinical benefit of TK inhibitors combined with chemotherapy; clearly the tumour target protein profile should be evaluated in different phases of treatment as well as at diagnosis or after chemotherapy or at relapse.

Furthermore, we would underline the importance of targets expression study in ME as in all rare pediatric tumors with a poor prognosis in order to identify alternative 
therapeutic strategies. We also propose regular molecular monitoring of the protein expression profile in tumor samples at reasonable time points during therapy administration. This may help taking decisions in drug selection including appropriate change or adjustment of chemotherapy.

\section{Abbreviations}

ME: Medulloepithelioma; PME: Peripheral Medulloepithelioma;

PDGFR: Platelet derived growth factor receptor; CNS: Central nervous system.

\section{Competing interests}

The authors declare that they have no competing interests.

\section{Authors' contributions}

MDDP conceptualized the report and designed the target studies addressing the patient treatment, collected clinical data, drafted the initial manuscript and approved the final manuscript as submitted. MADI reviewed and revised the manuscript, and approved the final manuscript as submitted. AG performed targeting studies on tumor specimens, critically reviewed the manuscript, and approved the final manuscript as submitted. AM critically reviewed the manuscript and approved the final manuscript as submitted. AC collected clinical data and approved the final manuscript as submitted. RC collected clinical data and approved the final manuscript as submitted. RB designed the target studies, performed and discussed targeting studies on tumor specimens, critically reviewed the manuscript and approved the final manuscript as submitted.

\section{Acknowledgment}

The authors would like to thank Professor Franco Locatelli for critically reviewing the paper

\section{Funding source}

No funding was secured for this study.

\section{Financial disclosure}

The authors have no financial relationships relevant to this article to disclose.

\section{Author details}

${ }^{1}$ Department of Pediatric Hematology/Oncology and Stem Cell Transplantation, Bambino Gesù Children's Hospital, Piazza Sant'Onofrio, 4 - 00165 Rome, Italy. ${ }^{2}$ Surgery Department, Bambino Gesù Children's Hospital, Rome, Italy. ${ }^{3}$ Pathology Department, Bambino Gesù Children's Hospital, Rome, Italy.

Received: 8 December 2013 Accepted: 16 February 2014

Published: 21 February 2014

\section{References}

1. Broughton WL, Zimmerman LE: A clinicopathological study of 56 cases of intraocular medulloepotheliomas. Am J Opthalmol 1978, 85(3):407-418.

2. Saunders T, Margo CE: Intraocular medulloepithelioma. Arch Pathol Lab Med 2012, 136(2):212-216.

3. Molloy PT, Yachnis AT, Rorke LB, Dattilo JJ, Needle MN, Millar WS, Goldwein JW, Sutton LN, Phillips PC: Central nervous system medulloepithelioma: a series of eight cases including two arising in the pons. J Neurosurg 1996, 84(3):430-436.

4. Milano GM, Cozza R, llari I, et al: High histologic and overall response to dose intensification of ifosfamide, carboplatin, and etoposide with cyclophosphamide, doxorubicin, and vincristine in patients with high-risk Ewing sarcoma family tumors: the Bambino Gesù Children's Hospital experience. Cancer 2006, 106(8):1838-1845.

5. Gm K, Young R, Re S: Primary neuroectodermal tumors of the ovary: a report of 25 cases. Am J Surg Pathol 1993, 17(8):764-778.

6. Figarella-Branger D, Gambarelli D, Perez-Castillo M, Gentet JC, Grisoli F, Pellissier JF: Ectopic intrapelvic medulloepithelioma: case report. Neuropathol Appl Neurobiol 1992, 18(4):408-414.

7. Bruggers CS, Welsh CT, Boyer RS, Byrne RS, Pysher TJ: Successful therapy in a child with a congenital peripheral medulloepithelioma and disruption of hindquarter development. J Pediatr Hematol Oncol 1999, 21(2):161-164.
8. Donner LR, Teshima I: Peripheral medulloepithelioma: an immunohistochemical, ultrastructural, and cytogenetic study of a rare, chemotherapy-sensitive, pediatric tumor. Am J Surg Pathol 2003, 27(7):1008-1012.

9. Nakamura Y, Becker LE, Mancer K, Gillespie R: Peripheral medulloepithelioma. Acta Neuropathol 1982, 57(2-3):137-142.

10. Ostman A: PDGF receptors-mediators of autocrine tumor growth and regulators of tumor vasculature and stroma. Cytokine Growth Factor Rev 2004, 15(4):275-286.

11. Gan HK, Burgess AW, Clayton AH, Scott AM: Targeting of a conformationally exposed, tumor-specific epitope of EGFR as a strategy for cancer therapy. Cancer Res 2012, 72(12):2924-2930.

12. Galeano F, Tomaselli S, Locatelli F, Gallo A: A-to-I RNA editing: the "ADAR" side of human cancer. Semin Cell Dev Biol 2012, 23(3):244-250.

13. Escudier B, Eisen T, Stadler WM, Szczylik C, Oudard S, Staehler M, Negrier S, Chevreau C, Desai AA, Rolland F, Demkow T, Hutson TE, Gore M, Anderson S, Hofilena G, Shan M, Pena C, Lathia C, Bukowski RM: Sorafenib for treatment of renal cell carcinoma: Final efficacy and safety results of the phase III treatment approaches in renal cancer global evaluation trial. J Clin Oncol 2009, 27(20):3312-3318.

14. Wang ZG, Zhang GF, Wu JC, Jia MK: Adjuvant therapy for hepatocellular carcinoma: Current situation and prospect. Drug Discov Ther 2013, 7(4):137--143.

15. Haraldsdottir S, Shah MH: An update on clinical trials of targeted therapies in thyroid cancer. Curr Opin Oncol 2014, 26:36-44.

16. Yang F, van Meter TE, Buettner R, Hedvat M, Liang W, Kowolik CM, Mepani N, Mirosevich J, Nam S, Chen MY, Tye G, Kirschbaum M, Jove R: Sorafenib inhibits signal transducer and activator of transcription 3 signaling associated with growth arrest and apoptosis of medulloblastomas. Mol Cancer Ther 2008, 7(11):3519-3526.

17. Wang CH, Hsu TR, Wong TT, Chang KP: Efficacy of temozolomide for recurrent embryonal brain tumors in children. Childs Nerv Syst 2009, 25(5):535-541.

18. Bomgaars LR, Bernstein M, Krailo M, Kadota R, Das S, Chen Z, Adamson PC Blaney SM: Phase II trial of irinotecan in children with refractory solid tumors: a Children's Oncology Group Study. J Clin Oncol 2007, 25(29):4622-4627.

doi:10.1186/1479-5876-12-49

Cite this article as: De Pasquale et al: Peripheral medulloepithelioma: a rare tumor with a potential target therapy. Journal of Translational Medicine 2014 12:49.

\section{Submit your next manuscript to BioMed Central and take full advantage of:}

- Convenient online submission

- Thorough peer review

- No space constraints or color figure charges

- Immediate publication on acceptance

- Inclusion in PubMed, CAS, Scopus and Google Scholar

- Research which is freely available for redistribution 